\title{
Does physical exercise improve ADL capacities in people over 65 years with moderate or severe dementia hospitalized in an acute psychiatric setting? A multisite randomized clinical trial
}

\author{
Elisabeth Bürge, ${ }_{1 \dagger}$ André Berchtold, ${ }^{2}$ Christine Maupetit, ${ }^{3}$ Nathalie M.-P. Bourquin, ${ }^{4}$ \\ Armin von Gunten, ${ }^{5}$ Daniel Ducraux, ${ }^{5}$ Serge Zumbach, ${ }^{6}$ Anne Peeters ${ }^{7}$ \\ and Nicolas Kuhne 8
}

\author{
${ }^{1}$ Department of Physical Therapy, HES-SO University of Applied Sciences and Arts Western Switzerland, Geneva, Switzerland \\ ${ }^{2}$ Institute of Social Sciences \& NCCR LIVES, University of Lausanne, Lausanne, Switzerland \\ ${ }^{3}$ Department of Nursing, HES-SO University of Applied Sciences and Arts Western Switzerland, Geneva, Switzerland \\ ${ }^{4}$ Research Institute, HES-SO University of Applied Sciences and Arts Western Switzerland, Geneva, Switzerland \\ ${ }^{5}$ Department of Psychiatry, Service of Old Age Psychiatry, Lausanne University Hospital, Lausanne, Switzerland \\ ${ }^{6}$ Department of Psychiatry and Psychotherapy for the elderly, Mental Health Network Fribourg (RFSM), Marsens, Switzerland \\ ${ }^{7}$ Grand Hôpital de Charleroi, Charleroi, Belgium \\ ${ }^{8}$ Department of Occupational Therapy, HES-SO University of Applied Sciences and Arts Western Switzerland, Lausanne, Switzerland
}

Background: Several studies on the effect of physical exercise on activities of daily living (ADL) for people with dementia exist; yet, data concerning the specific context of acute psychiatric hospitals remain scant. This study measured the effect of a physical exercise program on ADL scores in patients with moderate to severe dementia hospitalized in an acute psychiatric ward.

Methods: A multicenter clinical trial was conducted in five Swiss and Belgian psychiatric hospitals. Participants were randomly allocated to either an experimental group (EG) or a control group (CG). Members of the EG received 20 physical exercise sessions (strengthening, balance, and walking) over a fourweek period while members of the CG participated in social interaction sessions of equivalent duration and frequency, but without physical exercise. The effect of exercise on ADL was measured by comparing scores of the Barthel Index and the Functional Independence Measure in the EG and CG before and after the intervention, and two weeks later.

Results: Hundred and sixty patients completed the program. Characteristics of participants of both groups were similar at the inception of the study. The mean ADL score of EG decreased slightly over time, whereas that of the CG significantly decreased compared to initial scores. Overall differences between groups were not significant; however, significant differences were found for mobility-related items.

Conclusions: ADL scores in elderly with moderate to severe dementia deteriorate during acute psychiatric hospitalization. An exercise program delays the loss of mobility but does not have a significant impact on overall ADL scores.

Key words: dementia, activities of daily living (ADL), physical activity, exercise, behavioral and psychological symptoms of dementia (BPSD)

Correspondence should be addressed to: Nicolas Kuhne, OT, PhD, Professor UAS, University of Applied Sciences Western Switzerland, Occupational Therapy Department, Haute école de travail social et de la santé - EESP, Ch. des Abeilles 14, 1010 Lausanne, Switzerland. Phone: +41 7636930 74; Fax:+41 2165162 88. Email: nkuhne@gmail.com. Received 30 Nov 2015; revision requested 28 Jan 2016; revised version received 5 Aug 2016; accepted 16 Aug 2016. First published online 10 November 2016.

Trial number: ISRCTN86442281B Effects of a physical exercise program on daily living activities in dementia.

Trial register: ISRCTN Registry, c/o BioMed Central, Floor 6, 236 Grays Inn Road London, WC1X 8HB, United Kingdom.

†ied June 8, 2015.

\section{Introduction}

Dementia currently affects 24.3 million people, with 4.6 million new cases every year worldwide (Ferri et al., 2005). Dementia is characterized by a decline of cognitive functions and by non-cognitive behavioral and psychological symptoms (Cerejeira et al., 2012). Dementia is a major cause of functional dependence in people over 65 years and one of the main 
contributors to institutionalization (Luppa et al., 2010).

While patients suffering from dementia are frequently admitted to acute hospital wards because of Behavioral and Psychological Symptoms of Dementia (BPSD), independence in ADL is a major issue for the future of patients since a positive relationship exists between BPSD, level of independence in ADL, and caregiver burden (Miyamoto et al., 2010).

Forbes et al. (2008) conducted a systematic review on the effectiveness of physical activity programs in managing or improving cognition or function in people with dementia. They found insufficient evidence to either recommend physical activity programs, or advocate against their use. The review of Bürge et al. (2012) showed only weak evidence regarding the impact of exercise programs on ADL among patients with moderate or severe dementia. Rao et al. (2014) concluded, in their systematic review, that aerobic and strengthening exercises do improve physical performance and may help improve independence in ADL in people with Alzheimer disease. The most important benefit pertains to cognition and mobility. Walking exercise and habits seems to have a significant impact on cognitive function and independence (Kemoun et al., 2010; Venturelli et al., 2011; Winchester et al., 2013). Moreover, dementiarelated gait changes are not associated with motor disorders only but also with central misprocessing of information (Beauchet et al., 2008). Increasing evidence links alterations in executive function and attention to gait disturbances (Camicioli et al., 1997; Yogev-Seligmann et al., 2008). These studies support the hypothesis that the decline in ADL capacities in patients with dementia may be due not only to disease progression but also to physical inactivity (Littbrand et al., 2009).

Most of the investigations of the effect of exercise on ADL were conducted in nursing homes (Francese et al., 1997; Stevens and Killeen, 2006; Rolland et al., 2007) or in the community (Kwak et al., 2008; Steinberg et al., 2009). Only a few studies were conducted in acute psychiatric wards (Littbrand et al., 2009). As BPSD concern a significant number of people with dementia and represent a risk factor for acute care hospitalization, a better knowledge about the feasibility and effects of exercise programs in an acute care setting is relevant.

The aim of our study was to identify the impact of an exercise program on independence in ADL in patients with moderate or severe dementia hospitalized for BPSD. We hypothesized that a four-week program of daily exercise, accompanied by music and including strengthening, walking, and balance training:

1. can be carried out in an acute psychiatric ward;

2. is effective in maintaining or improving ADL capacities (maintaining or improving the scores of the Barthel Index (BI) and Functional Independence Measure (FIM)) in patients with moderate and severe dementia, as opposed to the control group (CG).

Additionally, we wanted to explore the influence of several independent variables (age, gender, medication, type and severity of dementia, comorbidities, body mass index, nutritional status, depression, BPSD) on the outcomes of the program.

\section{Methods}

\section{Design}

We conducted a randomized controlled trial in the French-speaking parts of Switzerland (four hospitals) and Belgium (one hospital). Patients were included in either the experimental group (EG, exercise program) or the CG (social program) by an independent person, using a computergenerated table of random numbers in blocks of 6. Notice that we included hospitals as a between-group factor in statistical analyses, but this factor was never significant, showing no difference between hospitals.

Patients were included if they were hospitalized in an acute psychogeriatric setting, had a diagnosis of dementia according to CIM-10, a Clinical Dementia Rating (CDR) of stage $\geq 2$ (moderate to severe dementia) and were able to walk at least $6 \mathrm{~m}$, if necessary with walking aids or assistance from one person. Patients were not included if the exercise program might put them at risk, if their life expectancy was estimated at less than four weeks, if they suffered from musculoskeletal conditions that did not permit the application of the exercise program, or if they had a recent diagnosis of hemiplegia. Exclusion criteria during the study were refusal to participate in the study - whether expressed by patients or their legal guardians, somatic or psychiatric deterioration requiring continuous or intensive care incompatible with the continuation of the study and, discharge or death of the patient.

ADL capacities, the primary outcome, was assessed before the intervention, after the intervention and two weeks later using the BI with ten items for a total score between 0 and 20 (Collin et al., 1988), and the FIM with 18 items for a total score between 7 and 126 (Keith et al., 1987). 


\section{Sample size}

For the sample size calculation (Type I error: 5\%, power: $80 \%$ ), we expected a strong correlation (0.95) between the three ADL measurements. Based on the results of Littbrand et al.'s study (2009), we assumed a mean BI score of 13 and a standard deviation of 4.5. This assumption was reinforced by the study conducted by Hsieh et al. (2007) estimating the minimal clinically important difference of the BI to be 1.85 points. We estimated the effect size at 0.2 using a mean difference of two points between groups and a standard deviation of five in each group. The required sample size was 192 participants; we chose to increase it by approximately $20 \%$ - up to 240 patients (120 patients in each group) - because of the physical frailty of the study population.

\section{Data collection}

The following independent variables were controlled and examined for their association with independence in ADL and/or with different levels of program adherence:

Social status, age, gender, and last practiced profession were assessed through medical history taking.

Somatic comorbidities were classified according to the Charlson Index by anamnesis (Charlson et al., 1987). Psychogeriatric comorbidities were based on medical history and observation and classified into the following three clinical categories: (1) mood disturbance, (2) personality disturbance, (3) spectrum of psychosis.

History of falls: Falls registered during the year preceding the current hospitalization as well as their severity (hospitalization, fracture of femoral neck) were collected by asking the patient, his/her family, or principal caregiver (Büla et al., 2006).

Medication was classified into eight categories: Hypnotics, Tranquilizers, Neuroleptics, Antiepileptics, Anti-depressants, Anti-hypertensives, Anti-Parkinson drugs, others.

Type of dementia was established clinically according to the following criteria: the diagnosis of dementia and Alzheimer's disease was made according to ICD-10 (International Classification of Diseases). The diagnosis of other types of dementia was based on established sources: Lewy body dementia, Fronto-temporal dementia, Vascular dementia, and subcortical vascular dementia).

Dementia severity was assessed using the French version of the CDR scale (Ousset et al., 2003).

We used the French version of the Mini-Mental State Examination to define the cognitive level of participants (Folstein et al., 1975; Derouesné et al., 1999).
Depression was assessed by the French version of the Cornell Depression Scale in Dementia (Camus et al., 1995).

Behavior was assessed using the French version of the Neuropsychiatric Inventory, brief version (Kaufer et al., 2000; Michel et al., 2005).

Adherence rate to the exercise program was assessed by the health professionals in charge of the intervention and rated on four levels. If the mean participation at the 20 intervention sessions was $\geq 3$ adherence was high, a mean $\geq 2<3$ corresponded to intermediate adherence and a mean score of $<2$ corresponded to weak program adherence (Rolland et al., 2007). The raw number of sessions (out of 20) in which the patients participated was also considered.

Assessments took place during the first fortnight of the patients stay at the hospital and the intervention program during weeks 3 to 6 . The twoweek period preceding the inception of treatment was meant to allow patients to get used to the new environment and reach a medically more stable condition.

\section{Measure of ADL capacities}

The modified BI (Collin et al., 1988) and the FIM (Keith et al., 1987) were applied through informant-based interviews by trained research assistants who were blind to group allocation. A total BI score of 20 and a total FIM score of 126 means full independence. Both instruments are reliable and valid (Kidd et al., 1995).

\section{Interventions}

All hospitalized sample members in the participating centers received the usual care program. The program provided was interdisciplinary in nature and corresponded to Cohen-Mansfield's classification (2001). It covered sensory interventions, social contacts, group interactions, structuring of the day, medical and systemic interventions, and specific attitudes on the part of care staff.

The exercise program corresponded to the program proposed by Rolland et al. (2000; 2007). It covered strength, flexibility, walking, and balance training and has been used with nursing home residents with moderate to severe dementia. Exercise intensity (number, pace, and duration) was gradually increased and adapted to each participant (Rolland et al., 2000; Rolland et al., 2007). The need for individual adaptation is essential as persons with such conditions do not always reliably express exertion and need specific guidance to exercise at the targeted level ( $\mathrm{Yu}$ et al, 2006). The aim was to achieve moderate intensity, as recommended for elderly patients 
as the threshold to obtain significant functional outcome by Paterson and Warburton (2010). As proposed by Rolland, after stretching warmup, participants were encouraged to walk fast to reach moderate breathlessness but not exhaustion. Walking is an important part of the program as walking is one of the most usual physical exercise to prevent later-life cognitive decline (Fallahpour et al., 2015). It is also very flexible for group use. Exercises included squatting at different levels (or repeated stand-ups from a chair), lateral elevation of the legs in a standing position, and rising on the toes. Participants were asked to imitate simple flexibility exercises demonstrated by the therapist. According the program proposed by Rolland et al. (2000; 2007), music accompanied some part of the sessions (Mathews et al., 2001) as music is known to enhance the effects of exercise programs for this population (Ziv and Lidor, 2011) although it does not seem to have a direct short-term effect on cognition ( $\mathrm{Li}$ et al., 2015). The exercise program was conducted with groups of at most four participants, in order to guarantee security and to correspond to the aforementioned requirements. Physical therapists, occupational therapists, or psychomotor therapists - all were given oral and written instructions related to the program - conducted five exercise sessions per week, each session lasting 30 minutes. The absence of specific guidelines on program duration, as well as the average length of stay of patients with moderate to severe dementia observed in the participating hospitals, led to the choice of four weeks for the program's duration.

The patients of the CG participated in a social activity program (watching videos about different topics or playing together) led by nurses, nursing auxiliaries, or occupational therapists. The number and duration of the sessions was the same as those of the exercise program.

Both programs, physical exercise, and social activity were held outside the ward on the same time schedule.

\section{Ethics}

The study was approved by all the relevant ethics' committees (250/08-VD; 027-CER-FR).

\section{Data analysis}

The socio-demographic and health characteristics of EG and CG were compared using the $\chi^{2}$ test for categorical variables and the Student's $t$ test for continuous ones. The BI and FIM scales were described by computing for each group and each time the mean, standard deviation, and $95 \%$ confidence interval. Baseline (T0) values were compared between groups using the Student's $t$ test. Changes of the BI and FIM scales between T0 and T2 were assessed using a repeatedfactor ANOVA. Repeated-factor ANOVAs were also performed separately for each component of the BI and FIM scales and each group of patients. Finally, we compared the adherence level of each group to the intervention as well as the association between adherence and ADL performance. The type I error was set to $5 \%$ for all statistical computations.

\section{Results}

Figure 1 presents the number of examined and included patients.

The main reasons for non-inclusion were short hospital stay, immediate discharge from the hospital, or not meeting the criteria set for following the exercise program. Three-hundred patients could not be included because of short stay. More than 300 patients could not do exercise because exercise was too risky $(n=118)$, contraindicated $(n=91)$, or because their medical conditions required continuous or intensive care $(n=102)$ (Figure 1).

Sixty-five participants dropped out between the inclusion and the end of intervention because they left the hospital. Other reasons for dropping out were diverse (such as delirium, acute BPSD, refusal by the participant or by his/her family, fall with fracture, and death) and led to the loss of 35 additional participants.

At baseline (T0), before the intervention, the socio-demographic and health characteristics of both groups of sample patients were statistically similar (all $p$-values $>0.05$, Table 1 ). Consequently, the following ANOVAs were not controlled for significant factors.

Table 2 describes the changes of the BI and FIM measures among both group of patients. At baseline (T0), the difference between groups was not significant (Student's $t(158)=0.119, p=$ 0.860 for BI, $t(158)=0.641, p=0.522$ for FIM). Both measures declined between $\mathrm{T} 0$ and $\mathrm{T} 2$ as indicated by repeated-factor ANOVAs $(F(2,316)=$ $7.58, p=0.001$ for $\mathrm{BI}$, and $F(2,316)=6.27$, $p=0.002)$, and no difference was found between groups $(F(1,158)=1.47, p=0.227$ for $\mathrm{BI}$, and $F(1,158)=1.52, p=0.220$ for FIM). A lack of power is to be noticed, due both to an insufficient number of patients completing the whole study and to differences between groups on the BI and FIM scales lower than expected when computing the required sample size. Consequently, the achieved power is only $30 \%$. 


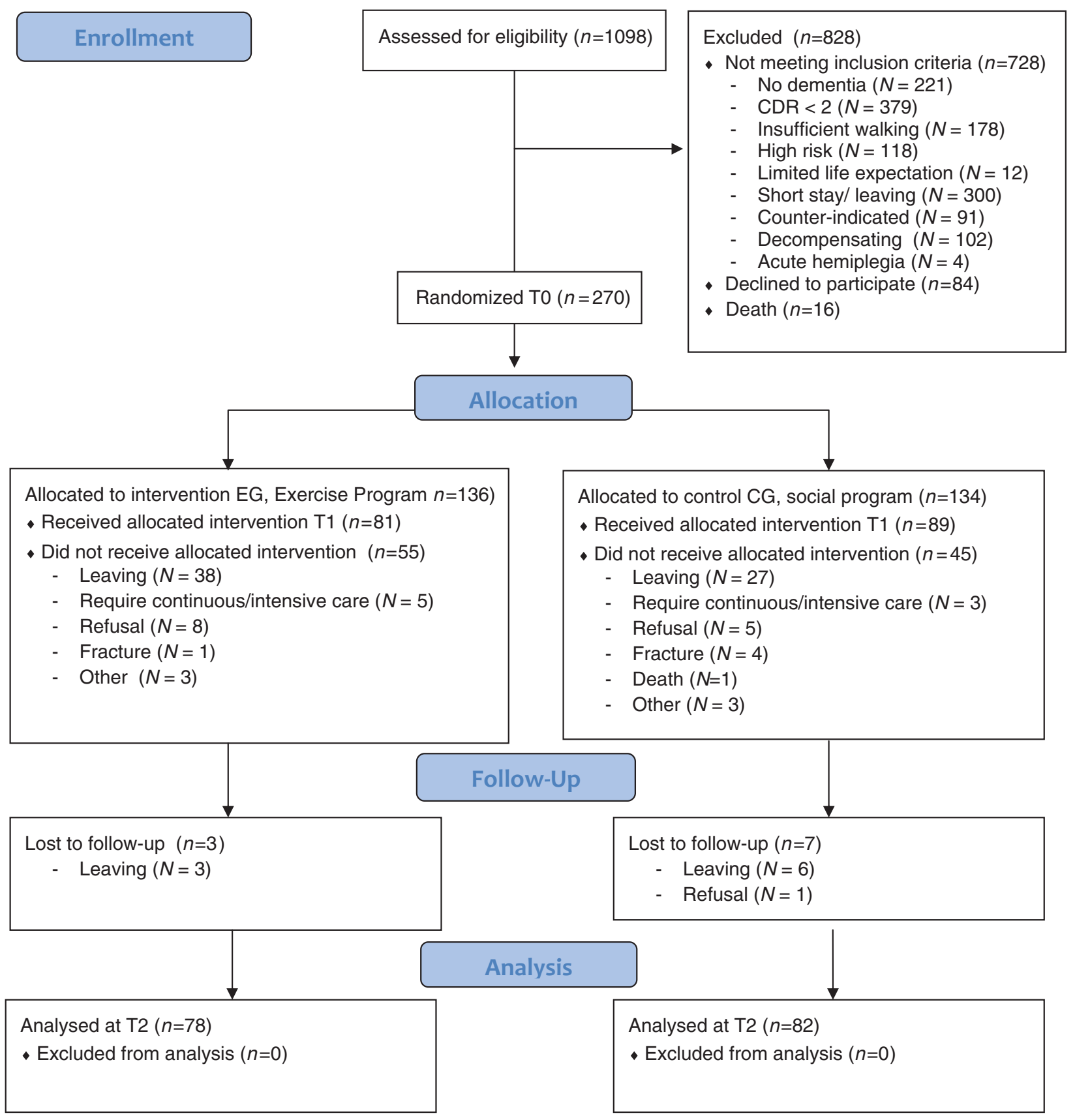

Figure 1. (Colour online) CONSORT 2010 flow diagram.

The BI scores of the different ADL measures showed that the EG's scores deteriorated in the bladder and bowel control areas, whereas the CG's scores decreased for the following four items: stairs, mobility, toilet use, and transfer (Table 3). The stairs item was the sole item for which the difference between groups was significant $(p=0.013)$.

The scores on the different components of the FIM show a deterioration in bowel in EG's results, but also in "Tub, Shower." Deterioration in mobility scores of the CG is also apparent.
It is to note that analyses presented in Tables 3 and 4 are only gross tendencies as these differences may be significant by chance.

Mean program adherence was similar in the EG (mean 2.04, sd 1.34) and CG (mean 2.02, sd1.05) groups $(t$-test $p$-value $=0.915)$. However, considering the classification of adherence into three groups (weak, intermediate, high), there was a significant difference between groups $\left(\chi^{2} p\right.$-value $=0.004)$. The percentage of patients with high adherence was larger in the EG than in the CG 
Table 1. Baseline (To) characteristics of participants who completed the whole study from To to T2 $(n=160)$. The last columns provide the $p$-value for the comparison of both groups of patients ( $\chi^{2}$ for categorical variables and $t$-test for continuous variables)

\begin{tabular}{|c|c|c|c|}
\hline & $\mathrm{EG}(n=78)$ & $\mathrm{CG}(n=82)$ & $p$-VALUE \\
\hline Age [years] mean (SD) & $81.7(7.7)$ & $81.1(7.7)$ & 0.642 \\
\hline Gender (female) & $48.7 \%$ & $53.7 \%$ & 0.532 \\
\hline \multicolumn{4}{|l|}{ Living situation before hospitalization: } \\
\hline Alone at home & $38.0 \%$ & $38.8 \%$ & \\
\hline With family at home & $40.8 \%$ & $45.0 \%$ & 0.727 \\
\hline In nursing home & $21.1 \%$ & $16.3 \%$ & \\
\hline $\mathrm{BMI}$, mean (SD) & $24.6(5.1)$ & $24.6(4.6)$ & 0.994 \\
\hline Level of albumin, $[\mathrm{g} / \mathrm{L}]$, mean $(\mathrm{SD})$ & $39.0(6,0)$ & $39.9(7.1)$ & 0.419 \\
\hline Level of protein, $[\mathrm{g} / \mathrm{L}]$, mean $(\mathrm{SD})$ & $65.3(6.3)$ & $65.9(8.4)$ & 0.643 \\
\hline \multicolumn{4}{|l|}{ Dementia: } \\
\hline Alzheimer disease & $51.3 \%$ & $54.5 \%$ & \\
\hline Lewy body dementia & $1.3 \%$ & $5.2 \%$ & \\
\hline Fronto-temporal & $3.9 \%$ & $1.3 \%$ & \\
\hline Vascular dementia & $2.6 \%$ & $5.2 \%$ & \\
\hline Subcortical & $1.3 \%$ & $1.3 \%$ & \\
\hline Mixt form & $23.7 \%$ & $11.7 \%$ & 0.308 \\
\hline Other & $15.8 \%$ & $20.8 \%$ & \\
\hline $\mathrm{CDR}$ score $=3$ & $32.1 \%$ & $34.1 \%$ & 0.778 \\
\hline MMSE, score $\leq 20$ & $84.2 \%$ & $78.1 \%$ & 0.394 \\
\hline Fall history & $39.2 \%$ & $34.6 \%$ & 0.559 \\
\hline Hospitalized for fall consequences & $15.1 \%$ & $17.9 \%$ & 0.634 \\
\hline $\operatorname{CSDD}[/ 38]$, score $>10$ & $35.1 \%$ & $38.5 \%$ & 0.661 \\
\hline NPI severity score $>2$ & $92.2 \%$ & $94.9 \%$ & 0.500 \\
\hline $\mathrm{CCI}$, mean (SD) & $6.7(1,7)$ & $6.6(2.1)$ & 0.807 \\
\hline Psychiatric comorbidities, mean (SD) & $1.8(1.0)$ & $2.1(1.0)$ & 0.064 \\
\hline Total number of medications, mean (SD) & $4.6(2.9)$ & $5.4(3.2)$ & 0.097 \\
\hline
\end{tabular}

$\mathrm{EG}=$ experimental group; $\mathrm{CG}=$ control group; $\mathrm{CDR}=$ clinical dementia rating; $\mathrm{MMSE}=$ Mini-Mental State Examination; CSDD = cornell scale for depression in dementia; NPI = neuropsychiatric inventory; CCI: Charlson comorbidity index.

Table 2. Description of ADL capacities among the patients $(n=160)$ who completed the whole study from To to $\mathrm{T} 2$

\begin{tabular}{|c|c|c|c|c|c|}
\hline \multirow[b]{2}{*}{ TIME } & \multirow[b]{2}{*}{ MEASURE } & \multicolumn{2}{|c|}{$\mathrm{EG}(n=78)$} & \multicolumn{2}{|c|}{$\mathrm{CG}(n=82)$} \\
\hline & & MEAN（SD） & $\mathrm{CI}_{95 \%}$ & MEAN（SD） & $\mathrm{CI}_{95 \%}$ \\
\hline \multirow[t]{2}{*}{ T0 } & $\mathrm{BI}$ & $13.44(4.424)$ & 12.44 to 14.43 & $13.32(4.088)$ & 12.42 to 14.22 \\
\hline & FIM & $79.74(21.554)$ & 74.88 to 84.60 & 77.67 (19.317) & 73.43 to 81.92 \\
\hline \multirow[t]{2}{*}{$\mathrm{T} 1$} & $\mathrm{BI}$ & $13.44(3.989)$ & 12.54 to 14.34 & $12.48(4.897)$ & 11.40 to 13.55 \\
\hline & FIM & $79.05(21.892)$ & 74.12 to 83.99 & 74.33 (22.489) & 69.39 to 79.27 \\
\hline \multirow[t]{2}{*}{$\mathrm{T} 2$} & $\mathrm{BI}$ & $12.77(4.623)$ & 11.73 to 13.81 & $11.59(5.137)$ & 10.46 to 12.71 \\
\hline & FIM & $75.90(24.107)$ & 70.46 to 81.33 & $71.29(23.599)$ & 66.11 to 76.48 \\
\hline
\end{tabular}

Legend: $\mathrm{T} 0=$ before the intervention, $\mathrm{T} 1=$ after the intervention, $\mathrm{T} 2$ : two weeks after the end of intervention; FIM = functional independence measure, $\mathrm{BI}=$ barthel index; $\mathrm{EG}=$ experimental group, $\mathrm{CG}=$ control group.

( $32.5 \%$ vs. $19.5 \%$ ), but the percentage of patients with weak adherence was also larger in the EG $(51.9 \%$ vs. $43.9 \%)$. On the other hand, there were more patients with intermediate adherence in the CG $(37.8 \%$ vs. $15.6 \%)$.

The measure of adherence, either raw or classified into three levels, is strongly related to the number of sessions in which the patients took part. The patients participated in average to 13.18 sessions out of a maximum of 20 (sd 6.83 ) in the EG group and to 13.80 sessions (sd 5.69 ) in the CG group, the difference being nonsignificant $(p=0.534)$. The correlation between raw adherence and the number of participating sessions was 0.86 for the EG group and 0.83 for the CG group, the difference between correlations 
Table 3. ANOVA for repeated measures comparing the three measurements (T0, T1, T2) of each component of the barthel index (BI)

\begin{tabular}{lcc}
\hline ITEMS OF B I & EG $(n=78)$ & CG $(n=82)$ \\
Feeding & 0.140 & 0.399 \\
Bathing & 0.057 & 0.783 \\
Bladder & 0.043 & 0.418 \\
Bowel & 0.019 & 0.413 \\
Mobility & 0.807 & 0.021 \\
Stairs & 0.779 & $<0.001$ \\
Dressing & 0.357 & 0.104 \\
Groom & 0.349 & 0.620 \\
Toilet & 0.539 & 0.011 \\
Transfer & 0.603 & 0.003 \\
\hline
\end{tabular}

Legend: For each test, we provide the $p$-value. In each situation, a significant $p$-value (two items for the EG (experimental group) and four items for the CG (control group) in light grey) indicates a worsening through time of the corresponding ADL.

being non-significant $(p=0.516)$. Similar results were obtained when replacing the raw measure of adherence by its three-levels version.

The comparison of the three categories of adherence with the factors presented in Table 1 showed that, for the EG, the type of dementia was significantly associated with adherence $(p=0.003)$; patients suffering from Alzheimer's disease being more adherent than other patients. Taking a higher number of medications was significantly associated with lower levels of adherence in the EG $(p=$ $0.012)$ and CG $(p=0.005)$ groups.

In the EG, patients with high program adherence showed a small increase in ADL performance between $\mathrm{T} 0$ and $\mathrm{T} 2$ (mean $\mathrm{BI}$ change score between T0 and T2 of 0.28), whereas patients with weak or intermediate adherence showed a decrease $(-1.08$ and -1.42 , respectively). However, due to intra-group variability, the difference was not significant $(F(2,74)=0.868 ; p=0.424)$. In the CG group, at all levels of adherence to the program, patients showed a decrease in their ADL performance $(-2.47$ for weak, -1.03 for intermediate, and -1.38 for high adherence, $F(2,79)=0.782 ; p=0.461)$.

\section{Discussion}

Summarizing the results, we observed that most of the participants experienced a decrease of independence in ADL, the CG experiencing a more important loss than the EG. The variability of individual scores and the fact that the sample was smaller than initially required may partly explain the absence of significant group differences.
Table 4. ANOVA for repeated measures comparing the three measurements (T0, T1, T2) of each component of the functional independence measure (FIM)

\begin{tabular}{lll}
\hline ITEMS OF FIM & $\begin{array}{l}\text { EG } \\
(n=78)\end{array}$ & $\begin{array}{l}\text { CG } \\
(n=82)\end{array}$ \\
\hdashline Eating & 0.085 & 0.177 \\
Grooming & 0.071 & 0.043 \\
Bathing & 0.325 & 0.011 \\
Dressing: upper body & 0.567 & 0.436 \\
Dressing: lower body & 0.744 & 0.568 \\
Toileting & 0.846 & 0.005 \\
Bladder management & 0.162 & 0.600 \\
iBowel management & 0.003 & 0.344 \\
Bed, chair, wheelchair & 0.609 & 0.029 \\
Toilet & 0.319 & 0.010 \\
Tub, shower & 0.018 & 0.885 \\
Walk (W), wheelchair (C), & 0.408 & 0.011 \\
$\quad$ or both & & \\
Stairs & 0.965 & 0.006 \\
Comprehension: A & 0.302 & 0.063 \\
$\quad$ (auditory) V (visual) B & & \\
$\quad$ (both) & & \\
Expression: V (vocal) N & 0.010 & 0.022 \\
$\quad$ (non-vocal) B (both) & & 0.633 \\
Social interaction & 0.130 & 0.330 \\
Problem solving & 0.571 & 0.268 \\
Memory & 0.436 & \\
\hline & &
\end{tabular}

Legend: For each test, we provide the $p$-value. In each situation, a significant $p$-value (three items for the EG (experimental group) and eight items for the CG (control group) in light grey) indicates a worsening through time of the corresponding ADL.

Interestingly however, the EG performed better on mobility items compared to the CG. These differences are coherent. The exercise program was centered on mobility, i.e. its main component walking and, for those patients who were able to manage stairs, climbing and descending stairs. These results confirm the findings of earlier studies indicating that exercise can improve mobility, including for people with dementia (Kemoun et al., 2010; Venturelli et al., 2011; Winchester et al., 2013). Developing clinical programs to improve mobility of these patients could be efficient.

By contrast, the difference between the two groups for items related to continence is surprising. According to literature, timed and prompted voiding seems to be the most effective measure for patients with moderate or severe dementia (Yap and Tan, 2006).

Various hypotheses, based on randomly selected adherence report sheets, may explain the mean low participation rate in the programs. The intensity of the program was high. Patients were supposed to follow the program five times per week during four weeks. For several patients, this frequency was 
too high. Specifically, with regard to the exercise program, some patients were not accustomed to exercise before hospitalization; the physical frailty of some others was too important and they were unable to exercise five times per week. Moreover, some of the patients did not understand the request of health professionals when the latter invited them to join the exercise group. When they were asked to join the program, some patients refused to leave the ward, where they felt more secure. When professionals did convince them to participate and they came into the exercise room with them, they did not always understand the professionals' instructions. Sometimes they did not understand the meaning of exercise. Patients with moderate to severe dementia do not have an intrinsic motivation to participate in an exercise program. Consequently, the health professionals who led these groups were faced with multiple challenges: they had to win the active participation of patients in the program, support them when necessary, adapt exercise intensity individually, maintain the participants' motivation over the 20 sessions, as well as guarantee the security of all patients in the group.

According to this study, the following patient profile was associated with good program adherence: diagnosis of Alzheimer disease, small number of medications, and living at home, alone or with family members. However, variations in ADL performance are expected since ADL loss in Alzheimer's disease patients varies widely (Arrighi et al., 2013). As observed by Rolland et al. (2007), general adherence rate was low; however, patients with good adherence experienced less of a decrease in their ADL performance.

The results also show that adherence differs significantly for specific subgroups (e.g. patients with $\mathrm{AD}$, or taking fewer medications). In clinical settings, an optimal intervention should therefore focus primarily on these patients. The program was proposed to all patients, but not all patients could participate in an optimal manner.

The duration of the programs raises some concerns. It has to have at least some duration in order to be effective; moreover, its organization has to take into account the limited average length of stay. Our choice for a $2+4$ week scheme is probably sub-optimal in regular clinical contexts. It was chosen in order to allow patients to reach a more stable condition prior to the intervention, but led to the loss of precious time for the intervention. Indeed, the duration of the intervention (four weeks) was a compromise. In order to be effective for most patients, such programs should begin as soon as possible after admission, in order to maximize their impact before discharge. Various types of health professionals led program sessions. They generally found the programs adapted to the acute hospital context and expressed appreciation for both EG and CG programs.

Based on the results of this study, and on the fact that the program was suited to only one in four of the admitted patients and completed by less than one in six, we would suggest, in order to increase adherence to an exercise regimen, integrating mobility and physical exercise in everyday activities, whenever the opportunity arises - for example, stimulating patients with moderate or severe dementia hospitalized in acute hospital wards to walk to the dining room. Physical exercise could then be seen as the by-product of a more meaningful activity. Preserving mobility has an important impact on the burden experienced by caregivers and loss of ADL capacities increases the patient's risk of being admitted to a nursing home (Miyamoto et al., 2010). Integrated exercise could also be more easily continued when patients return to their previous place of residence. However, it must be noted that individual stimulation lacks the positive elements provided by music as well as by the group.

The most important limitation of the study is the lack of power due both to an insufficient number of participants completing the study and to lower differences between groups than expected. Another limitation is the high dropout rate of more than one in three patients, even if very few participants actually refused to continue once enrolled in the study. Most of the dropouts - one in four sampled patients - resulted from discharge from the hospital, occurring before the end of the program. While the duration of the program, four weeks, was seen as a compromise between the duration required for an effective program and the drop out risk, it was actually too long with regard to the length of stay of a significant proportion of the participants.

As physical exercise is known to have an impact on mobility as well as non-mobility-related functions, we chose an overall $\mathrm{ADL}$ assessment. In doing so, we weakened the measurable impact of exercise on mobility scores.

In conclusion, ADL capacities do deteriorate during acute hospital stays in patients with moderate or severe dementia. An exercise program conducted by various health professionals with a focus on strength, balance, and walking for patients with moderate to severe dementia turned out to be feasible in the various acute psychogeriatric wards included in the study, but its clinical interest seems limited to patients who enjoy participating and who have less severe deficits and higher length of stay. Regular stimulation of walking could contribute to 
delaying the deterioration of mobility that occurs in these patients during acute hospitalization. Future research should try to explore other ways to foster regular physical exercise for patients with moderate or severe dementia in this specific setting.

\section{Conflict of interest}

None.

\section{Description of authors' roles}

Elisabeth Bürge formulated the research questions, designed the study, carried it out, analyzed the data, and wrote the paper. André Berchtold contributed to formulate the research questions, to design the study, analyzed the data, co-wrote the statistical and the results parts of the paper, and revised the paper. Christine Maupetit contributed to formulate the research questions, to design the study, to carry it out, and revised the paper. Nathalie Bourquin contributed to carry it out, and revised the paper. Armin von Gunten contributed to formulate the research questions, to design the study, to carry the study out, and co-wrote the paper. Daniel Ducraux contributed to carry the study out, and read the paper critically. Serge Zubach contributed to formulate the research questions, to design the study, to carry the study out, and read the paper critically. Anne Peeters contributed to carry the study out, and revised the paper. Nicolas Kuhne contributed to formulate the research questions, to design the study, to carry it out, to analyze the data, and co-wrote the paper.

\section{References}

Arrighi, H. M., Gélinas, I., McLaughlin, T. P., Buchanan, J. and Gauthier, S. (2013). Longitudinal changes in functional disability in Alzheimer's disease patients. International Psychogeriatrics, 25, 929-937. doi: 10.1017/S1041610212002360.

Beauchet, O., Allali, G., Berrut, G., Hommet, C., Dubost, V. and Assal, F. (2008). Gait analysis in demented subjects: interests and perspectives. Neuropsychiatric Disease and Treatment, 4, 155-160.

Büla, C., Rochat, S. and Dunand, G. (2006). Chutes et personnes âgées. Primary Care, 6, 200-203.

Bürge, E., Kuhne, N., Berchtold, A., Maupetit, C. and von Gunten, A. (2012). Impact of physical activity on activity of daily living in moderate to severe dementia: a critical review. European Review of Aging and Physical Activity, 9, 27-39. doi: 10.1007/s11556-011-0092-y.

Camicioli, R., Howieson, D., Lehman, S. and Kaye, J. (1997). Talking while walking the effect of a dual task in aging and Alzheimer's disease. Neurology, 48, 955-958. doi: 10.1212/WNL.48.4.955.

Camus, V., Schmitt, L. Ousset, P. J. and Micas, $M$. (1995). Depression and dementia: contribution to the French validation of 2 depression scales: the cornell scale for depression in dementia and the dementia mood assessment scale. Encephale, 21, 201-208.

Cerejeira, J., Lagarto, L. and Mukaetova-Ladinska, E. B. (2012). Behavioral and psychological symptoms of dementia. Frontiers in Neurology, 3, 73. doi: 10.3389/fneur.2012.00073.

Charlson, M. E., Pompei, P., Ales, K. L. and MacKenzie, C. R. (1987). A new method of classifying prognostic comorbidity in longitudinal studies: development and validation. Fournal of chronic diseases, 40, 373-383.

Cohen-Mansfield, J. (2001). Nonpharmacologic interventions for inappropriate behaviors in dementia. American fournal of Geriatric Psychiatry, 9, 361-381.

Collin, C., Wade, D. T., Davies, S. and Horne, V. (1988). The barthel ADL index: a reliability study. International Disability Studies, 10, 61-63.

Derouesné, C., Poitreneau, J., Hugonot, L., Kalafat, M., Dubois, B. and Laurent, B. (1999). Le mini mental state examination (MMS). Un outil pratique pour l'évaluation de l'état cognitif des patients par le clinicien. Presse Médicale, 28, 1141-1148.

Fallahpour, M., Borell, L., Luborsky, M. and Nygård, L. (2015). Leisure-activity participation to prevent later-life cognitive decline: a systematic review. Scandinavian fournal of Occupational Therapy, 0, 1-36. http://doi.org/10.3109/11038128.2015.1102320.

Ferri, C. P. et al. (2005). Global prevalence of dementia: a Delphi consensus study. Lancet, 366, 2112-2117.

Folstein, M. F., Folstein, S. E. and McHugh, P. R. (1975). "Mini-mental state", practical method for grading the cognitive state of patients for the clinician. Fournal of Psychiatric Research, 12, 189-198.

Forbes, D., Forbes, S., Morgan, D. G., Markle-Reid, M., Wood, J. and Culum, I. (2008). Physical activity programs for persons with dementia. The Cochrane Database of Systematic Reviews, CD006489. doi: 10.1002/14651858.CD006489.

Francese, T., Sorrell, J. and Butler, F. R. (1997). The effects of regular exercise on muscle strength and functional abilities of late stage Alzheimer's residents. American fournal of Alzheimers Disease and Other Dementias, 12, 122-127.

Hsieh, Y. W., Wang, C. H., Wu, S. C., Chen, P. C., Sheu, C. F. and Hsieh, C. (2007). Establishing the minimal clinically important difference of the Barthel Index in stroke patients. Neurorehabilitation and Neural Repair, 21, 233-238.

Kaufer, D. I. et al. (2000). Validation of the NPI-Q, a brief clinical form of the Neuropsychiatric Inventory. The fournal of Neuropsychiatry and Clinical Neurosciences, 12, 233-239.

Keith, R. A., Granger, C. V., Hamilton, B. B. and Sherwin, F. S. (1987). The functional independence measure: a new tool for rehabilitation. Advances in Clinical Rehabilitation, 1, 6-18.

Kemoun, G. et al. (2010). Effects of a physical training programme on cognitive function and walking efficiency in 
elderly persons with dementia. Dementia and Geriatric Cognitive Disorders, 29, 109-114.

Kidd, D. et al. (1995). The functional independence measure: a comparative validity and reliability study. Disability and Rehabilitation, 17, 10-14.

Kwak, Y. S., Um, S. Y., Son, T. G. and Kim, D. J. (2008). Effect of regular exercise on senile dementia patients. International fournal of Sports Medicine, 29, 471-474.

Li, H.-C., Wang, H.-H., Chou, F.-H. and Chen, K.-M. (2015). The effect of music therapy on cognitive functioning among older adults: a systematic review and meta-analysis. Fournal of the American Medical Directors Association, 16, 71-77. doi: 10.1016/j.jamda.2014.10.004.

Littbrand, H., Lundin-Olsson, L., Gustafson, Y. and Rosendahl, E. (2009). The effect of a high-intensity functional exercise program on activities of daily living: a randomized controlled trial in residential care facilities. Fournal of the American Geriatrics Society, 57, 1741-1749. doi: $10.1111 /$ j.1532-5415.

Luppa, M., Luck, T., Weyerer, S., König, H. H., Brähler, E. and Riedel-Heller, S. G. (2010). Rediction of institutionalization in the elderly. A systematic review. Age and Ageing, 39, 31-38. doi: 10.1093/ageing/afp202.

Mathews, R. M., Clair, A. A. and Kosloski, K. (2001). Keeping the beat: use of rhythmic music during exercise activities for the elderly with dementia. American fournal of Alzheimer's Disease and Other Dementias, 16, 377-380.

Michel, E. et al. (2005). Validation of the short version of the Neuropsychiatric inventory [NPI-Q]. La Revue de Gériatrie, 30, 385-390.

Miyamoto, Y., Tachimori, H. and Ito, H. (2010). Formal caregiver burden in dementia: impact of behavioral and psychological symptoms of dementia and activities of daily living. Geriatric Nursing, 31, 246-253. doi: 10.1016/j.gerinurse.2010.01.002.

Ousset, P. J., Andrieu, S., Reynish, E., Puel, M. and Vellas, B. (2003). Clinical evaluation of dementia in a cohort of 358 patients with the French version of the clinical dementia rating (CDR) scale. La Revue de Médecine Interne, 24, 283s-287s.

Paterson, D. H. and Warburton, D. E. (2010). Physical activity and functional limitations in older adults: a systematic review related to Canada's physical activity guidelines. The International fournal of Behavioral Nutrition and Physical Activity, 7, 38. doi: 10.1186/1479-5868-7-38.
Rao, A. K., Chou, A., Bursley, B., Smulofsky, J. and Jezequel, J. (2014). Systematic review of the effects of exercise on activities of daily living in people with Alzheimer's disease. American fournal of Occupational Therapy, 68, 50-56. http://doi.org/10.5014/ajot.2014.009035.

Rolland, Y. et al. (2000). Feasibily of regular physical exercise for patients with moderate to severe Alzheimer disease. The fournal of Nutrition, Health $\mathcal{E}$ Aging, 4, 109-113.

Rolland, Y. et al. (2007). Exercise program for nursing home residents with Alzheimer's disease: a 1-year randomized, controlled trial. Fournal of the American Geriatrics Society, $55,158-165$.

Steinberg, M., Leoutsakos, J. M., Podewils, L. J. and Lyketsos, C. G. (2009). Evaluation of a home-based exercise program in the treatment of Alzheimer's disease: the maximizing independence in dementia (MIND) study. International fournal of Geriatric Psychiatry, 24, 680-685. doi: $10.1002 /$ gps. 2175 .

Stevens, J. and Killeen, M. (2006). A randomised controlled trial testing the impact of exercise on cognitive symptoms and disability of residents with dementia. Contemporary Nurse, 21, 32-40.

Venturelli, M., Scarsini, R. and Schena, F. (2011). Six-month walking program changes cognitive and adl performance in patients with alzheimer. American fournal of Alzheimer's Disease and Other Dementias, 5, 381-388. doi. org/10.1177/1533317511418956.

Winchester, J. et al. (2013). Walking stabilizes cognitive functioning in Alzheimer's disease (AD) across one year. Archives of Gerontology and Geriatrics, 56, 96-103.

Yap, P. and Tan, D. (2006). Urinary incontinence in dementia - a practical approach. Australian Family Physician, 35, 237-241.

Yogev-Seligmann, G., Hausdorff, J. M. and Giladi, N. (2008). The role of executive function and attention in gait. Movement Disorders, 3, 329-342.

Yu, F., Kolanowski, A. M., Strumpf, N. E. and Eslinger, P. J. (2006). Improving cognition and function through exercise intervention in Alzheimer's disease. Fournal of Nursing Scholarship, 38, 358-365.

Ziv, G. and Lidor, R. (2011). Music, exercise performance, and adherence in clinical populations and in the elderly: a review. Fournal of Clinical Sport Psychology, 5, $1-23$. 